\title{
Water Flow Generator: Innovating Water Faucet Use
}

\section{Prof. Bala Maheswaran, Northeastern University}

Bala Maheswaran, PhD Northeastern University 367 Snell Engineering Center Boston, MA 02115

Mr. Yifan Guo

Alejandro Hervella

Mr. Aleksei Pavlov

Minh Duc Dinh 


\title{
Water Flow Generator: Innovating Water Faucet Use
}

\author{
Alejandro Hervella, Yifan Guo, Minh Duc Dinh, Aleksei Pavlov, and Bala Maheswaran \\ College of Engineering \\ Northeastern University
}

In the past decades, people are using more electricity in their daily life; therefore, it is crucial to develop methods to produce energy from day-to-day doings. Considering our average daily use, the device was designed to harness energy from water flow using fundamental physics concepts. Hydroelectric power is considered as one of the cleanest alternative energies but is still not widely and efficiently used by most of today's energy enterprises. This paper presents a water flow generator that produces electrical energy from the day-to-day water use at home. The flow generator design is a micro view of the hydroelectric power plant combined with the idea of using rechargeable and energy storing devices. This will help to generate electrical energy to support some of the devices used daily by people. The prototype generator is based on the minuscule hydropower generator and will take advantage of water flow from faucets.

This study explores the practicality of converting water flow energy into electrical energy with a mini generator design using simple physics concepts. Effectively harnessing water flow could recover a significant amount of usable electrical energy from simple, everyday actions with minimal impact to daily operation. This design shows promise for the future of off-grid energy generation. The skills learned from this fluid physics based project were invaluable, as research, design, trial and error, as well as technical writing are all important experiences within engineering education. This generator not only provides scientists and researchers with more valuable information about alternative energy, but is also capable of educating the everyday person about the basics of engineering and physics, as well as the importance of renewable resources from daily activities.

\section{Introduction}

Water is becoming more important and more valuable in today's society mainly because the total amount of the water supply existing on Earth has been decreasing for decades. Daily use of water, which supports human life, for example, to drink or clean, makes up a huge proportion of the water needed from the nature environment. Faucets are one of the useful inventions created by the engineers, which has already helped and keep helping humans with their daily life. However, the truth is, the more faucets humans are using, the more water will flow from human consumption. How to innovate the use of water into energy from the faucets we use has become the most urgent problems today ${ }^{1-6}$.

Traditional energy today is harnessed using fossil fuels such as coal power plants, which will produce an excessive amount of emissions and pollutions to the environment. In the year of 2007, 48 percent of the electricity produced by the United States came from the traditional power plants, 22 percent which were from natural gas. Instead of using traditional ways to produce electricity, scientists and engineers have been working hard for years to find an alternative way to generate energy. Solar energy, which takes power from the sun using solar panels, is one of the cleanest ways to produce energy. Nonetheless, the high cost of maintenance and initial set up proved that this kind of energy source needs to be more efficient and improved.

Hydropower energy appears to be the cleanest and best alternative energy today, which uses gravity and the pressure of the running water to spin the turbine that connects to the generator to produce energy. However, this kind of power plant is not widely and efficiently used today ${ }^{1}$.

The idea of the water flow generator combines the concept of gravitational force and the turbines of a power plant; our design reduces the size of the hydropower plant and at the same time uses the water flow from the faucet as the source of 
energy. Inside the generator is a rotor and turbine along with a circuit inside, which can transfer the electricity produced by the generator from $\mathrm{AC}$ to DC. The maximum voltage and power output will be $5 \mathrm{~V}$ and 10 Watts.

\section{Methodology}

Our initial design focused on housing all the components in the device attached directly to the faucet. However, after reevaluating the size of the design, we decided to separate the output energy source into a power bank that could be used to charge a phone or portable battery. Figure 1 shows the final model of the water generator to the left, and the grey power bank station to the right.

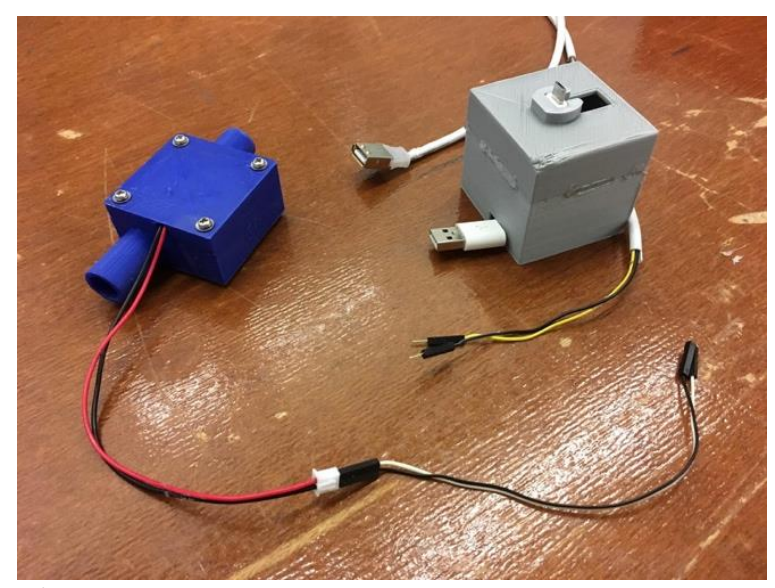

Figure 1: Generator components

The power bank is equipped with a female USB extender (wired to the generator) so that the user can open the box and use the desired cable of choice to charge a phone, battery, or other device. The water generator can be installed before the water runs through the faucet, on the nozzle of the faucet, or after the water flows down to the sink. Out of the three installations, the water generator attached to the nozzle of the faucet is the most practical as well as the most efficient to harness electricity due to the assistance of gravity as the water falls.

The turbine which has inner round pieces of metal attached on top of the generator is perfectly held in the case. The turbine generator is then placed under the running water of a standard faucet with a diameter of $31.8 \pm 0.5$ millimeters. As the water passes through the nozzle, the turbine rotates. The pieces of metal in the turbine magnetically induces an electric field via the copper coil to generate electricity. Finally, the whole generator has a flexible elastic piece at the nozzle entrance which can be affixed to almost all common faucets, making the design universal for all sinks.

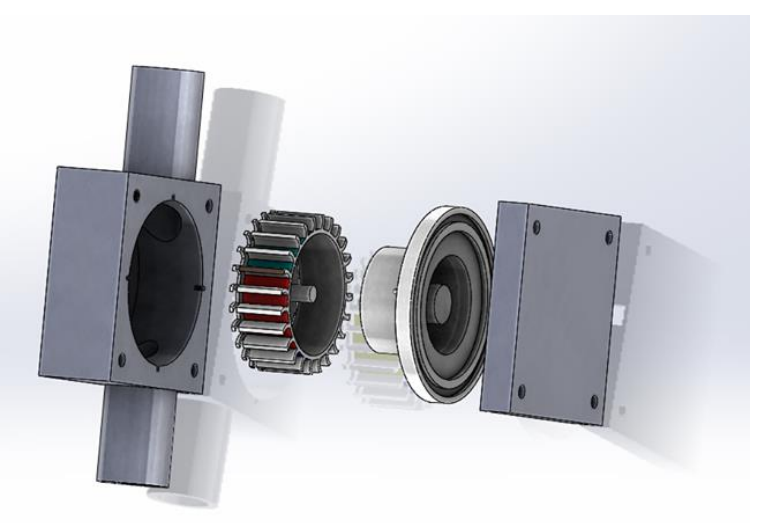

Figure 2: Exploded View of 3D Printed Parts

\section{Results and Discussion}

After receiving our purchased mini generator via the postal mail, it was very apparent that the device was meant for water hoses and needed a significant amount of pressure to get the turbine spinning. No matter what faucet was used, the turbine would not spin and could not generate a voltage due to the small hole at the nozzle of the device. As a result, when 3D parts were finally printed, it was decided to widen the hole in order for the turbine to have enough surface contact with the faucet water to spin. Figure 4 shows the nozzle hole of the purchased part, and figure 5 shows the 3D printed encasing which has a substantially wider nozzle.

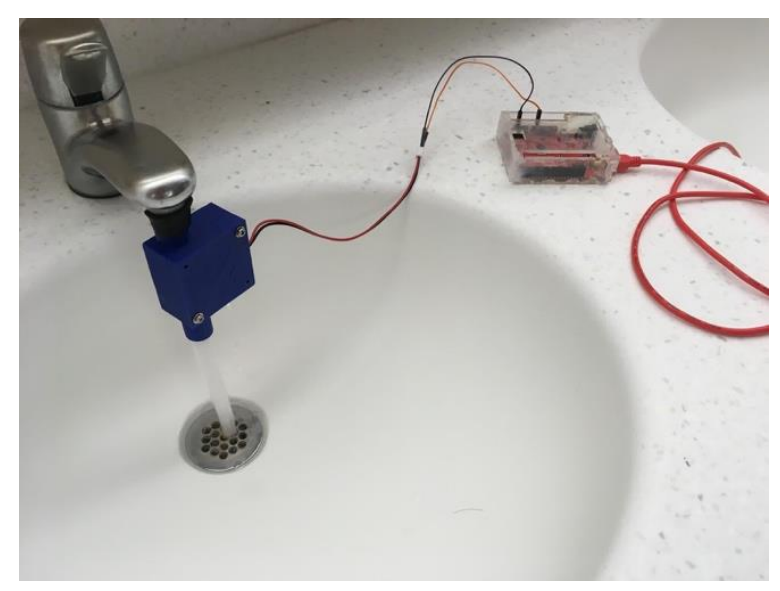


Figure 3: Prototype in Action with Running Faucet

After printing the parts, taking apart the purchased model, and installing the 5 volts 10 watts generator that it came with. After multiple trials and errors, widening the nozzle on the 3D printed device, and measuring voltage using the Sparkfun Redboard and Arduino programming software, three distinct sets of data from three different faucets were recorded.

Figure 6 was conducted with a strong faucet, figure 7 was with a medium faucet, and figure 8 was with a weak faucet.

This distinction is accurate since the power of the faucet is directly correlated with the voltage generated, with figure 1 and the most powerful faucet reaching just below 5 volts, and figure 3 with the weakest faucet reaching an average of under 3 volts.

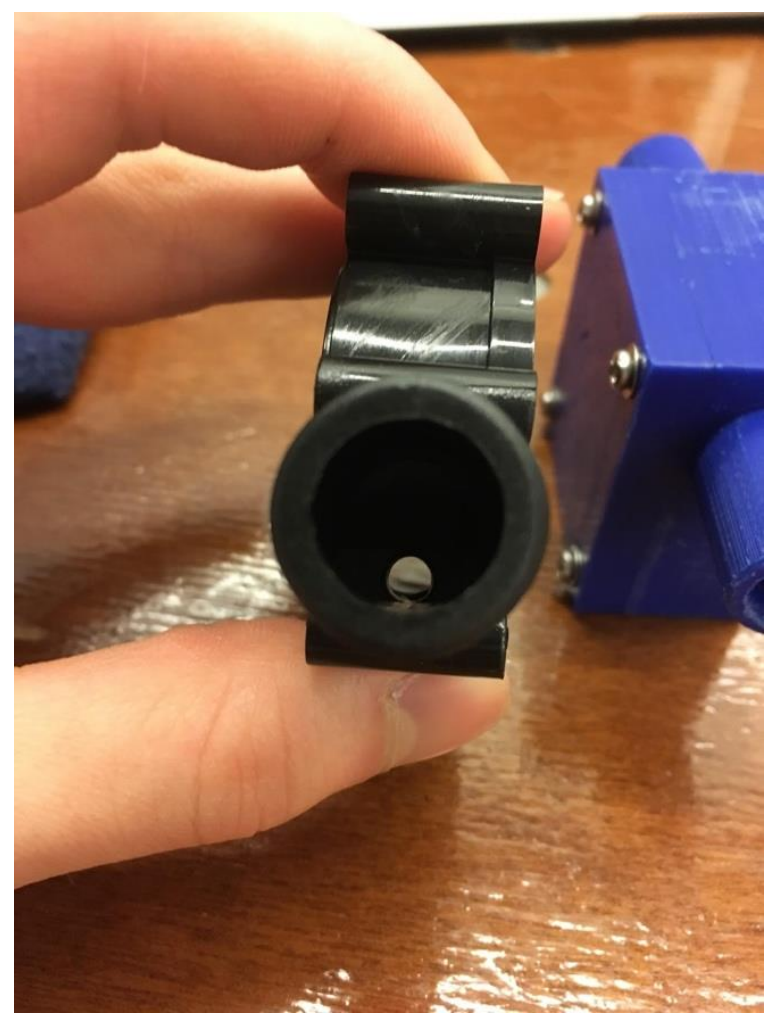

Figure 4: Nozzle

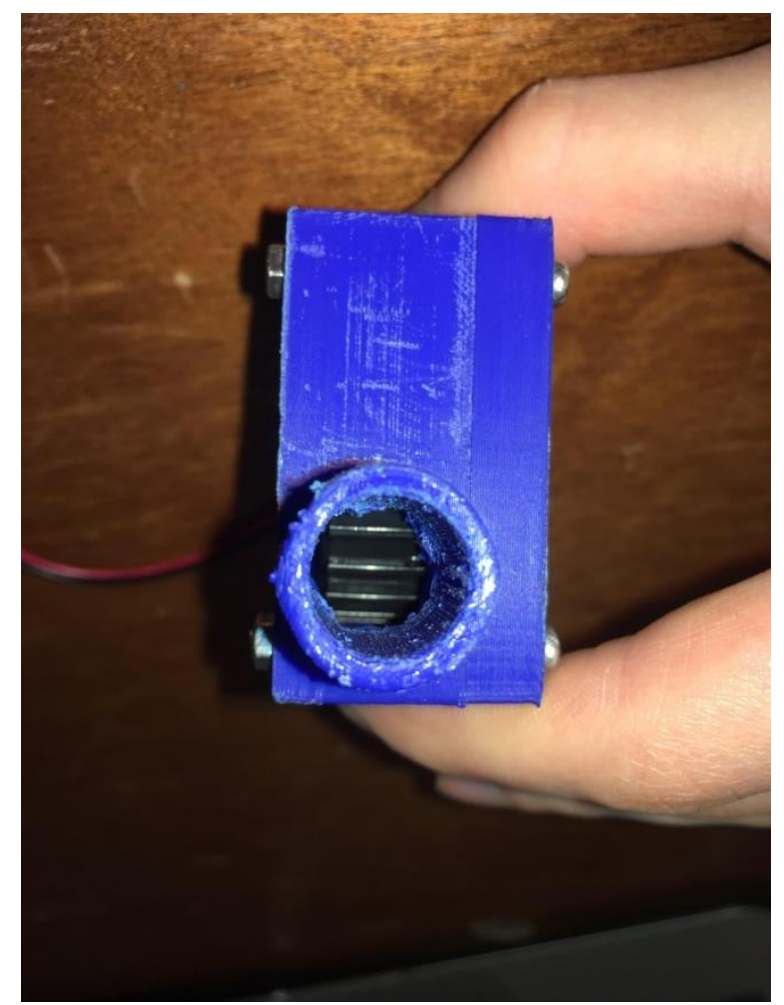

Figure 5: 3D Printed Nozzle

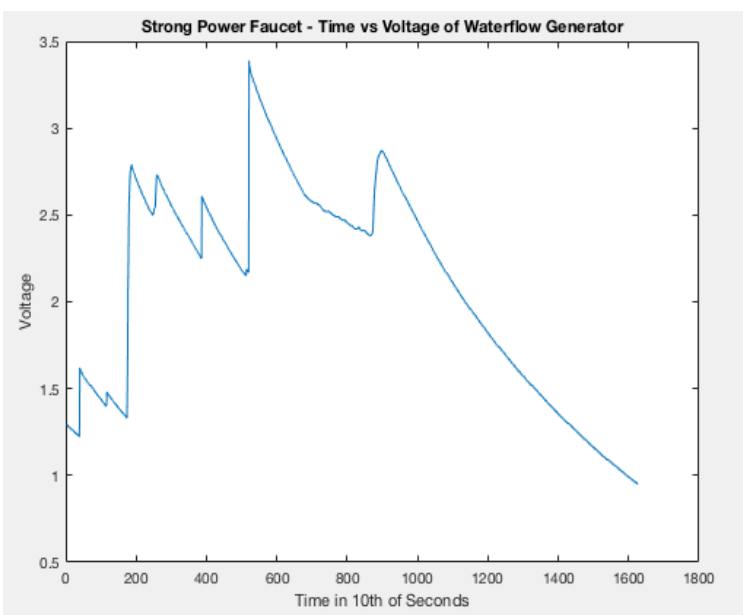

Figure 6: Strong Power Faucet Data 


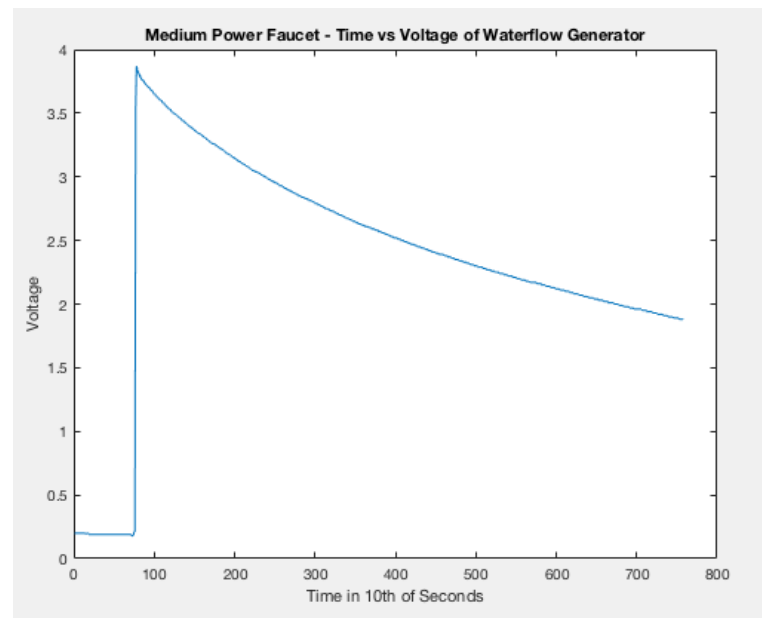

Figure 7: Medium Power Faucet Data

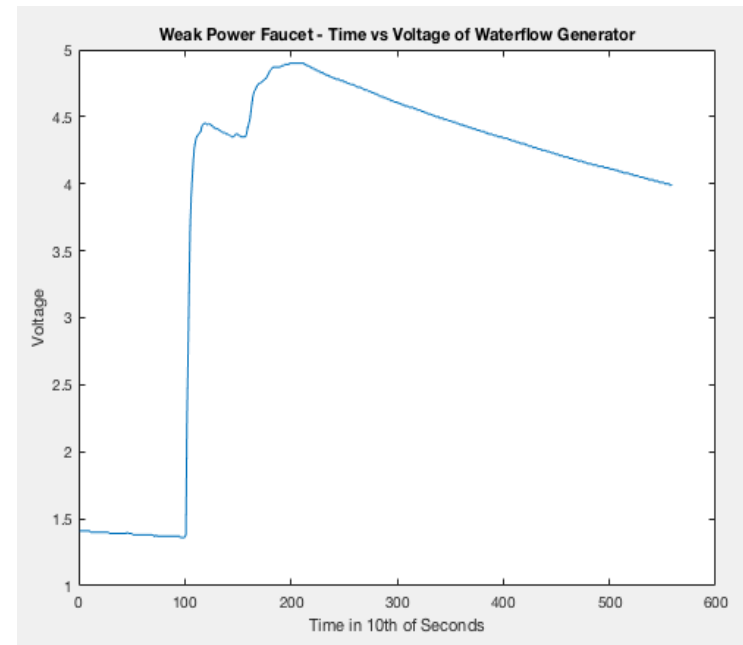

Figure 8: Weak Power Faucet Data

Nonetheless, it can be seen that after pulling away from the faucets, there is still a significant voltage that steadily decreases, occurring at around 20, 15, and 100 seconds in figures 6, 7, and 8, respectively. We realized that this is due to the fact that the generator's circuit board has a capacitor installed, which in turn is actually a very helpful feature. The addition of a capacitor allows for voltage to be generated even if there are short breaks when the faucet is not running, so that charging devices will continue to receive power during these short intervals without running water.

\section{Analysis}

The amount of energy that could be saved from utilizing this design was calculated using estimates and statistics obtained from the US government population census website. Using an estimated population of 327 million people ${ }^{3}$, with each using on average 13 gallons a day, about 4.25 billion gallons of water runs from faucets on a daily basis in the US. The data shows that water flow from typical faucets generates an average of about 3.5 volts $^{2}$. Knowing that our generator is designed to be 10 watts with a max of 5 volts, and using the equation

$$
\text { volts } \times \text { amps }=\text { watts }
$$

the generator should have a current of 2 amps. Therefore, the average wattage generated with our device was

$$
\begin{gathered}
3.5 \text { volts } \times 2.0 \text { amps }=7.0 \text { watts } \\
=0.0070 \text { kilowatts }
\end{gathered}
$$

Furthermore, government data also shows that most faucets use about 1.50 gallons per minute. That means that with the $4.25 \times 10^{6}$ gallons used daily from faucets, there is approximately $6.38 \times 10^{9}$ minutes $\left(1.06 \times 10^{8}\right.$ hours $)$ of faucet use on a daily basis in the US. If theoretically our device was installed on just half of all the faucets used in the U.S.A, the amount of energy yielded would be:

\section{$1.06 \times 10^{8} / 2$ hours $\times 0.0070$ kilowatts $=$ $3.72 \times 10^{5} \mathrm{kWh}$.}

That means within the course of one day, the US could generate about $3.72 \times 10^{5}$ kilowatt-hours with half of the faucets available. Multiplied out to the time span of a year (365 days), the US could save over $1.35 \times 10^{8}$ kilowatt hours. This is extremely significant and would be shared throughout the population on an individual level and household.

\section{Conclusion and Implications}

This engineering product brings a new way of using renewable energy in our everyday activities. During market research we figured out that some prototypes have been already existing. However, we decided to stay with our idea and try to innovate an existing model. 
After making the working prototype, there were several new advantages to this innovative project. The design works with both powerful and weak faucets and water fountains. In addition, the rubber nozzle connector makes our device very flexible and universal in size and simplifies the attachment of the device to the faucet. Furthermore, the generator that we made was very cheap in manufacturing, even in the prototype stage. Therefore, the mass production of this design would be extremely cost effective.

Upon completing the design, future developments were speculated, including integrating the generator inside the faucet and increasing the efficiency of the system.

To sum up, this project was a successful combination of simplicity, outstanding engineering and design, and it has high commercial potential in the future.

\section{Acknowledgments}

The authors would like to express their sincere thanks to Dr. Donald Goldthwaite and the First year Engineering learning and Innovation center lab Assistants for their support. We'd also like to thank Erin Dooley for technical and teaching support.

\section{References}

1) U.S. Department of the Interior, \& USGS. (2016, December 2). Hydroelectric power: How it works. Retrieved April 19, 2018, fromhttps://water.usgs.gov/edu/hyhowworks $\underline{. h t m l}$
2) U.S. Department of the Interior, \& USGS. (2016, December 02). Water Questions \& Answers How much water does the average person use at home per day? Retrieved April 19, 2018, from https://water.usgs.gov/edu/qa-homepercapita.html

3) U.S. Census Bureau. (2018, April 19). U.S. and World Population Clock Tell us what you think. Retrieved April 19, 2018, from https://www.census.gov/popclock/

4) Maupin, M.A., Kenny, J.F., Hutson, S.S., Lovelace, J.K., Barber, N.L., and Linsey, K.S., 2014, Estimated use of water in the United States in 2010: U.S. Geological Survey Circular 1405, 56 p., https://dx.doi.org/10.3133/cir1405

5) $10 \mathrm{~W}$ Water Turbine Generator Micro Hydroelectric Power Generator DIY LED Power Charging Tool DC 5V: Automotive. (n.d.). Retrieved April 19, 2018, from https://www.amazon.com/Water-TurbineGenerator-HydroelectricCharging/dp/B01N63BGCX

6) Gaines, C. M. (2012, July 06). Water Infographic: Alarming Statistics About Wasted Water in Our Communities. Retrieved April 19, 2018, from https://www.watercache.com/blog/2012/07/ water-infographic-with-water-wastestatistics-from-leaky-pipes 


\section{Appendix 1: Data Collection via Matlab and Arduino Software}

Matlab Code (for figures 6, 7, \& 8):

s1 = importdata('faucet1.txt');

s2 = importdata('faucet2.txt');

s3 = importdata('faucet3.txt');

b1 $=[1: 1: 559]^{\prime} ;$

b2 $=[1: 1: 758]^{\prime}$

b3 $=[1: 1: 1627]^{\prime} ;$

figure(1);

$\operatorname{plot}(\mathrm{x}, \mathrm{y})$;

title('Time vs Voltage of Waterflow

Generator')

xlabel('Time in 10th of Seconds')

ylabel('Voltage');

figure(2);

$\operatorname{plot}(x 2, y 2)$;

title('Time vs Voltage of Waterflow

Generator')

xlabel('Time in 10th of Seconds')

ylabel('Voltage');

figure(3);

plot(x3, y3);

title('Time vs Voltage of Waterflow

Generator')

xlabel('Time in 10th of Seconds')

ylabel('Voltage');

figure(4);

plot(b1, s1);

title('Weak Power Faucet - Time vs Voltage

of Waterflow Generator')
xlabel('Time in 10th of Seconds')

ylabel('Voltage');

figure(5);

plot(b2, s2);

title('Medium Power Faucet - Time vs

Voltage of Waterflow Generator')

xlabel('Time in 10th of Seconds')

ylabel('Voltage');

figure(6);

plot(b3, s3);

title('Strong Power Faucet - Time vs Voltage

of Waterflow Generator')

xlabel('Time in 10th of Seconds')

ylabel('Voltage');

Arduino Code for Voltage Reading (in serial

monitor):

void setup() \{

Serial.begin(9600);

\}

void loop() \{

int data = analogRead(A3);

delay (100) // prints every 100

milliseconds (10th of a second)

float volts $=$ data * $(5.0 / 1023.0) ; \quad / /$

received range for 5 volt port is 0 to 1023

Serial.println(volts); 